\title{
Mechanical Analysis and Safety Evaluation of Double-Packer string in a horizontal well
}

\author{
Yinping Cao, Hao Li and Yihua Dou \\ Mechanical Engineering College, Xi'an Shiyou University
}

\begin{abstract}
During the hydraulic compression packer setting process, axial stress of the string will be generated, which may affect the safety of the string. In this paper, the double-packer string in a horizontal well is taken as the object. Considering the two packers are seated separately, on the basis of Lame formula, generalized Hooke' $s$ law and bar deformation theory, the equations of axial force and axial stress of the string from the packers, and the deformation of the string without constraint from packers are deduced under wellhead pressure. The results show that, when packer 1 is sealed with wellhead pressure as $\mathbf{1 0}$ MPa, the axial stress generated on the string between the bending section and packer 1 is $49.94 \mathrm{MPa}$. When packer 2 is sealed with wellhead pressure as $15 \mathrm{MPa}$, the axial stress generated on the string between the bending section and the packer 2 is $58.05 \mathrm{MPa}$ The axial stress of the string between packer 1 and packer 2 is reduced to $15.08 \mathrm{MPa}$ and the total axial stress of the string between the bending section and packer 2 is $107.96 \mathrm{MPa}$. It can be seen that packer 2 increases the axial stress on the string between the packer and the bending section when it is seated and causes the axial stress of the string between packer 1 and packer 2 reduced. Thus, the dangerous point locates between packer 2 and the curved section of the string. The axial stress near the bottom of the string is reduced and the string tends to be safe. The results can be used to provide references for the selection of horizontal string to ensure its safety.
\end{abstract}

Keywords - Mechanical Analysis; string; Double-Packer; Horizontal Well

\section{INTRODUCTION}

In the process of separate-layer water injection and separate-layer fracturing, the downhole string needs to be connected with multiple packers to achieve separate-layer production of oil and gas well [1]. Due to the effect of the packer sealing process, the string will deform when the downhole packer is sealed. The axial stress will occur in the string because its deformation is constrained by the sealed packer, which can affect the safety of the string. In order to ensure the safety of the downhole string and tools, it is necessary to analyze the force and length of the strings before and after packer is sealed.

In the 1960s, Lubinski first analyzed the force and deformation of a string with a single packer. He studied the axial deformation of the single-packer string under the four effects based on Hooke's law and the spiral buckling theory of string [2]. In the 1980s, Hammerlindl further studied the stress and deformation of double-packer string [3 4]. In 2001, Qindao Li systematically analyzed and discussed the force and deformation of single-packer string [5 6]. There are also many domestic and foreign experts and scholars studied the packer string later, but most of them only focus on the packer string in vertical wells and intubation packers, which is relatively simple [7 10]. In 2014, Zhanguo Lv conducted the mechanical analysis of the multi- hydraulic compression packer string in a horizontal well. He only researched the influence on the force and length of the string when each packer was sealed, while ignoring the interaction between the various packers [11]. Considering the shortage of existing research, in this paper the double-packer string and the hydraulic compression packers in a horizontal well are taken as the object. Considering the influence of the later sealed packer on the force and length of the string and previous sealed packers, the equations of the axial force and axial stress of the string from the packer under wellhead pressure are deduced. Besides that, the deformation of the string without packer is also deduced.

\section{RESEARCH SCOPE AND BASIC ASSUMPTIONS}

\section{A. Research scope}

In this paper, double-packer string in a horizontal well, hydraulic compression packers and a uniform string are taken as the object.

\section{B. Basic assumptions}

The basic assumptions adopted are: The well is filled with liquid; ignoring the weight of string; ignoring the buckling deformation of string; when the packer is seated, it is pushed by successive steps; the packer is named from the bottom of the well, i.e. packer 1 , packer 2, respectively.

Based on the assumptions, the diagram of double-packer string in a horizontal well is shown in Fig. $1 . h$ is the depth of the wellhead to the horizontal section. The distance between the end of the bending section and packer 1 is $L$; the distance between packer 1 and packer 2 is $L_{1}$, called the first section string; and the distance between packer 2 and the end of the bending section is $L_{2}$, called the second section string.

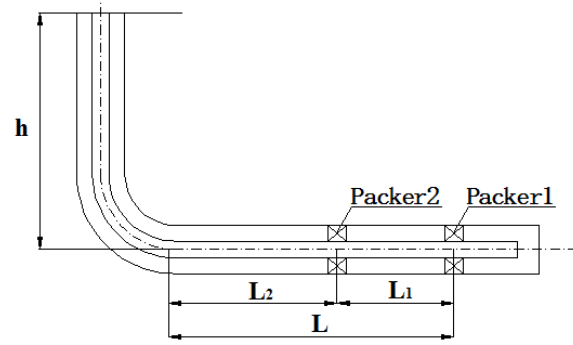

Fig.1 The diagram of double - packer string in a horizontal well 


\section{MECHANICAL ANALYSIS OF STRING WHEN PACKER 1 IS SEATED}

During the process of packer 1 setting, a smaller ball is put from the wellhead into the balloon of packer 1 and then wellhead pressure $p_{1}$ is applied. From the Lame formula, the radial stress and circumferential stress in the horizontal section string from the wellhead pressure are [12]:

$$
\begin{gathered}
\sigma_{1 r}=\frac{\left(p_{1 i} d^{2}-p_{o} D^{2}\right)}{D^{2}-d^{2}}-\frac{\left(p_{1 i}-p_{o}\right) D^{2} d^{2}}{\left(D^{2}-d^{2}\right) d_{i}^{2}} \\
\sigma_{1 \theta}=\frac{\left(p_{1 i} d^{2}-p_{o} D^{2}\right)}{D^{2}-d^{2}}+\frac{\left(p_{1 i}-p_{o}\right) D^{2} d^{2}}{\left(D^{2}-d^{2}\right) d_{i}^{2}}
\end{gathered}
$$

Where, $p_{0}$ is the outside pressure of the string at the bottom, $\mathrm{MPa} ; D$ is the outside diameter of the string, $\mathrm{m} ; d$ is the inside diameter of the string, $\mathrm{m} ; d_{i}$ is the diameter in an arbitrary place of the string, $\mathrm{m} ; p_{1 i}$ is the downhole pressure caused by wellhead pressure, which can be calculated as follows:

$$
p_{1 i}=p_{1}+\rho_{i} g h
$$

Where, $\rho_{i}$ is the density of the liquid in the string, $\mathrm{kg} / \mathrm{m}^{3}$.

Because of the above two stresses, the string will conduct radial deformation, which will arise axial deformation. According to the generalized Hooke 's law, the axial strain of the horizontal section string is [13]:

$$
\varepsilon_{1 z}=\frac{1}{E}\left[\sigma_{1 z}-\mu\left(\sigma_{1 r}+\sigma_{1 \theta}\right)\right]
$$

Where, $E$ is the elastic modulus of the string, $\mathrm{MPa} ; \mu$ is the Poisson's ratio of the string; $\sigma_{1 z}$ is the axial stress of horizontal section string, MPa.

The axial deformation is:

$$
\Delta L=\varepsilon_{1 z} L=\frac{L}{E}\left[\sigma_{1 z}-\mu\left(\sigma_{1 r}+\sigma_{1 \theta}\right)\right]
$$

Assuming that the horizontal section string has no constraint from packer 1 , that is, the string is not subjected to axial force, then the string will be free to deform. At this time the axial stress $\sigma_{1 z}$ of the string is zero.

Substituting zero in (5) for $\sigma_{1 z}$, we can obtain:

$$
\Delta L=\varepsilon_{1 z} L=-\frac{\mu\left(\sigma_{1 r}+\sigma_{1 \theta}\right) L}{E}=-\frac{2 \mu\left(p_{1 i} d^{2}-p_{0} D^{2}\right) L}{E\left(D^{2}-d^{2}\right)}
$$

Because of the bending friction and the packer 1 constrained each ends of the horizontal section string, the axial deformation of the horizontal section string was limited, so that the axial restraining force was generated on the string from the packer 1. This axial force makes the deformation of the string is zero, i.e. $\Delta L=0$.

Substituting zero in (5) for $\Delta L$, we can obtain:

$$
\sigma_{1 z}=\mu\left(\sigma_{1 r}+\sigma_{1 \theta}\right)=\frac{2 \mu\left(p_{1 i} d^{2}-p_{o} D^{2}\right)}{D^{2}-d^{2}}
$$

The corresponding axial force is:

$$
F_{1}=\sigma_{1 z} A_{s}=\frac{\pi}{2} \mu\left(p_{1 i} d^{2}-p_{o} D^{2}\right)
$$

Where, $A_{s}$ is the cross-sectional area of the string, $\mathrm{m}^{2}$.

\section{MECHANICAL ANALYSIS OF STRING WHEN PACKER 2 IS SEATED}

During the process of packer 2 setting, a larger ball is put from the wellhead into the balloon of the packer 2 and then the wellhead pressure $p_{2}$ is applied. Also, the radial stress $\sigma_{2 r}$ and circumferential stress $\sigma_{2} \theta$ in the second section string will be generated.

Because of the above two stresses, the second section string will conduct radial deformation, which will arise axial deformation. According to the generalized Hooke 's law, the axial strain of the second section string is:

$$
\varepsilon_{2 z}=\frac{1}{E}\left[\sigma_{2 z}-\mu\left(\sigma_{2 r}+\sigma_{2 \theta}\right)\right]
$$

Where, $\sigma_{2 z}$ is the axial stress of the second section string, $\mathrm{MPa}$.

The axial deformation is:

$$
\Delta L_{2}=\varepsilon_{2 z} L_{2}=\frac{L_{2}}{E}\left[\sigma_{2 z}-\mu\left(\sigma_{2 r}+\sigma_{2 \theta}\right)\right]
$$

Because of the bending friction and the packer 2 constrained each ends of the second section string, the axial deformation of the string was limited, so that the axial restraining force was generated on the string from the packer 2 . This axial force makes the deformation of the second section string is zero, i.e. $\Delta L_{2}=0$.

Substituting zero in (10) for $\Delta L_{2}$, we can obtain:

$$
\sigma_{2 z}=\mu\left(\sigma_{2 r}+\sigma_{2 \theta}\right)=\frac{2 \mu\left(p_{2 i} d^{2}-p_{o} D^{2}\right)}{D^{2}-d^{2}}
$$

Where, $p_{2 i}$ is the downhole pressure caused by wellhead pressure $p_{2}, \mathrm{MPa}$, which can be obtained when substituting $p_{2}$ in (3) for $p_{1}$.

The corresponding axial force is:

$$
F_{2}=\sigma_{2 z} A_{s}=\frac{\pi}{2} \mu\left(p_{2 i} d^{2}-p_{o} D^{2}\right)
$$

The horizontal section string with two packers is now simplified as a simple beam, as shown in Fig. 2. In the figure, the first constraint from left to right is the bending friction on the string, the second constraint is the packer 2 to the string, and the third constraint is the packer 1 . 


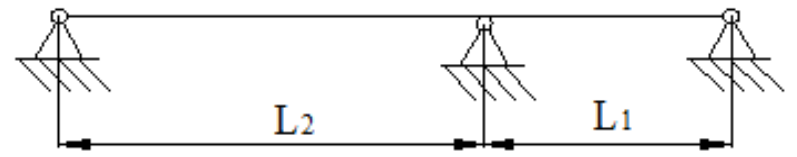

Fig.2 The simplified diagram of the horizontal segment packer string

If the constraints from the packers 1 and 2 are removed, the second section string will be free to deform under the action of the wellhead pressure $p_{2}$. At this time the axial stress $\sigma_{2 z}$ of the second section string is zero.

Substituting zero in (10) for $\sigma_{2 z}$, we can obtain:

$$
\Delta L_{2}=\varepsilon_{2 z} L_{2}=-\frac{\mu\left(\sigma_{2 r}+\sigma_{2 \theta}\right) L_{2}}{E}=-\frac{2 \mu\left(p_{2 i} d^{2}-p_{0} D^{2}\right) L_{2}}{E\left(D^{2}-d^{2}\right)}
$$

If the constraint from the intermediate packer 2 is removed without removing the constraint from the packer 1, the length of the horizontal section string will remain constant under two constraints from the bending section and the packer 1 when the wellhead pressure $p_{2}$ is applied, but the axial deformation will be generated on the second section string, assuming the deformation is shortening and the size is $\Delta L_{2}^{\prime}$, as a result the first section string will elongate, assuming the deformation is $\Delta L_{1}^{\prime}$.

From the above analysis, it can be seen that a "virtual axial force" which has the same size as $F_{2}$ and opposite to $F_{2}$ is generated on the position of packer 2 when the constraint from the packer 2 is removed. The force shortens the second section string and elongates the first section string, and the deformation is $\Delta L_{2}{ }^{\prime}$ and $\Delta L_{1}{ }^{\prime}$, respectively, as shown in Fig. 3.

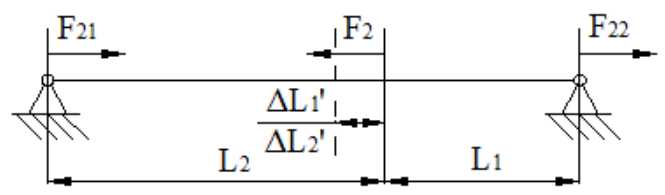

Fig.3 The diagram of removing the packer 2 constraint

In the figure above, the axial force $F_{21}$ is generated from the bending section, and the axial force $F_{22}$ is generated from the packer 1. Assuming the rightward axial force is positive, then by the static balance of the conditions, we can obtain:

$$
F_{21}-F_{2}+F_{22}=0
$$

Because of the above equation has one known force $F_{2}$ and two unknown forces $F_{21}$ and $F_{22}$, the simple beam structure is a statically indeterminate structure.

According to the above analysis, we can obtain the deformation coordination equation is:

$$
\Delta L_{1}{ }^{\prime}+\Delta L_{2}{ }^{\prime}=0
$$

And physical equation:

$$
\begin{gathered}
\Delta L_{1}{ }^{\prime}=\frac{F_{22} L_{1}}{E A_{s}} \\
\Delta L_{2}{ }^{\prime}=-\frac{F_{21} L_{2}}{E A_{s}}
\end{gathered}
$$

Substituting (16) and (17) into (15), we obtain:

$$
F_{22} L_{1}-F_{21} L_{2}=0
$$

Simultaneous (14) and (18), we can obtain:

$$
\begin{aligned}
& F_{21}=\frac{F_{2} L_{1}}{L_{1}+L_{2}} \\
& F_{22}=\frac{F_{2} L_{2}}{L_{1}+L_{2}}
\end{aligned}
$$

It can be seen that the axial restraining forces from the bending section and the packer 1 are $F_{21}$ and $F_{22}$ respectively, but all opposite to the direction shown in Fig. 3 when the constraint from the packer 2 is present.

From the above analysis, it can be seen that a leftward axial force is generated on the string from the packer 1 when the packer 2 is seated, so that the axial force generated on the horizontal section string from the packer 1 is changed. We can obtain:

$$
F_{1}{ }^{\prime}=F_{1}-F_{22}
$$

\section{SAFEty Evaluation}

It can be seen from the above analysis, when the two packers are sealed, the axial stress of the string between the end of the bending section and the packer 2 is:

$$
\sigma_{2 z}{ }^{\prime}=\sigma_{1 z}+\sigma_{2 z}
$$

The axial stress of the string between the packer 2 and packer 1 is:

$$
\sigma_{1 z}^{\prime}=\sigma_{1 z}-\frac{L_{2}}{L_{1}+L_{2}} \sigma_{2 z}
$$

Using the fourth strength theory of material mechanics to check:

$$
\sigma_{4}=\sqrt{\frac{1}{2}\left[\left(\sigma_{z}-\sigma_{\theta}\right)^{2}+\left(\sigma_{z}-\sigma_{r}\right)^{2}+\left(\sigma_{r}-\sigma_{\theta}\right)^{2}\right]}
$$

When check the first section string, the axial stress in (24) is obtained from (23), and the radial stress and the circumferential stress in (24) are obtained from (1) and (2), respectively. When check the second section string, the axial stress in (24) is obtained from (22), and the radial stress and the circumferential stress in (24) can be obtained by substituting $p_{2 i}$ in (1) and (2) for $p_{1 i}$. 


\section{CALCULATION EXAMPLE}

The depth $h$ of the horizontal well is $4000 \mathrm{~m}$, the distance $L_{2}$ between the end of the bending section and the packer 2 is $600 \mathrm{~m}$, the distance $L_{1}$ between the packers 1 and 2 is $400 \mathrm{~m}$, the outside diameter $D$ of the string is $88.9 \mathrm{~mm}$, the inside diameter $d$ of the string is $76 \mathrm{~mm}$, the Poisson's ratio $\mu$ is 0.3 , the elastic modulus $E$ is $2.1 \times 10^{11} \mathrm{~Pa}$, the permissible stress is $758 \mathrm{MPa}$, the completion fluid density is $1.2 \times 10^{3} \mathrm{~kg} / \mathrm{m}^{3}$, the pressure $p_{0}$ outside the string at the bottom is $20 \mathrm{MPa}$, the wellhead pressure when the packers 1 and 2 are seated is 10 $\mathrm{MPa}$ and $15 \mathrm{MPa}$, respectively.

\section{A. Calculations of force and deformation of string when the packer 1 is sealed}

From the (6), when there is no constraint from packer 1, the axial deformation of the horizontal section string is:

$$
\Delta L=-\frac{2 \mu\left(p_{1 i} d^{2}-p_{0} D^{2}\right) L}{E\left(D^{2}-d^{2}\right)}=-0.24 \mathrm{~m}
$$
is:

From the (7), the axial stress of the horizontal section string

$$
\sigma_{1 z}=\frac{2 \mu\left(p_{1 i} d^{2}-p_{o} D^{2}\right)}{D^{2}-d^{2}}=49.91 \mathrm{MPa}
$$

From the (8), the axial force of the horizontal section string is:

$$
F_{1}=\frac{\pi}{2} \mu\left(p_{1 i} d^{2}-p_{o} D^{2}\right)=83.38 \mathrm{KN}
$$

Direction to the right.

B. Calculations of force and deformation of string when the packer 2 is sealed

From the (13), when there are no constraints from packers 1 and 2, the axial deformation of the horizontal section string is:

$$
\Delta L_{2}=-\frac{2 \mu\left(p_{2 i} d^{2}-p_{0} D^{2}\right) L_{2}}{E\left(D^{2}-d^{2}\right)}=-0.17 \mathrm{~m}
$$

From the (11), the axial stress of the second section string is:

$$
\sigma_{2 z}=\frac{2 \mu\left(p_{2 i} d^{2}-p_{o} D^{2}\right)}{D^{2}-d^{2}}=58.05 \mathrm{MPa}
$$

From the (12), the axial force that is generated from the packer 2 is:

$$
F_{2}=\frac{\pi}{2} \mu\left(p_{2 i} d^{2}-p_{o} D^{2}\right)=96.99 \mathrm{KN}
$$

Direction to the right.

From the (20), the axial force that is generated from the packer 1 is:

Direction to the left.
From the (21), when the packer 2 is seated, the total axial force generated on the horizontal section string from the packer 1 is:

$$
F_{1}{ }^{\prime}=F_{1}-F_{22}=25.18 \mathrm{KN}
$$

\section{Strength check}

1) Strength check of the first section string

From the (1) and (2), the radial stress and the circumferential stress is:

$$
\sigma_{1 r}=-36.78 \mathrm{MPa}, \sigma_{1 \theta}=203.14 \mathrm{MPa}
$$

From the (23), the axial stress of the string is:

$$
\sigma_{1 z}{ }^{\prime}=15.08 \mathrm{MPa}
$$

From the (24), the equivalent stress of the string is:

$$
\sigma_{4}=218.65 \mathrm{MPa}
$$

It is less than the permissible stress of $758 \mathrm{Mpa}$, thus the first section string is safety.

\section{2) Strength check of the second section string}

From the (1) and (2), the radial stress and the circumferential stress is:

$$
\sigma_{2 r}=-38.99 \mathrm{MPa}, \sigma_{2 \theta}=232.49 \mathrm{MPa}
$$

From the (22), the axial stress of the string is:

$$
\sigma_{2 z}{ }^{\prime}=107.96 \mathrm{MPa}
$$

From the (24), the equivalent stress of the string is:

$$
\sigma_{4}=235.38 \mathrm{MPa}
$$

It is less than the permissible stress of $758 \mathrm{Mpa}$, thus the second section string is safety.

\section{CONCLUSIONS}

(1)During the hydraulic compression packer setting process, the radial stress and the circumferential stress on the string will be generated, resulting in axial deformation of the string is generated.

(2)Because of the packer constrains the string, limits the axial deformation of the string, the axial deformation is converted into the axial restraining force on the string from the packer, resulting in axial stress of the string is generated.

(3)The later sealed packer increases the axial stress on the string between the packer and the bending section when it is seated and causes the axial stress of the string between the packer and previous sealed packer reduced. Thus, the dangerous point locates between the later sealed packer and the curved section of the string. The axial stress near the bottom of the string is reduced and the string tends to be safe.

\section{ACKNOWLEDGMENT}

The paper is supported by Chinese National Natural Science Foundation (51404198) and Natural Science Foundation of Shaanxi Province, China (2014JQ7289). 


\section{REFERENCES}

[1] LiPing Zhang, Cen Gao, Qingjiang Gu. Technical Measures for Increasing Production of Oilfield [J]. China Petroleum and Chemical Standards and Quality, 2013, 39(8):96-96.

[2] Lubinski A, Althouse W S. Helical Buckling of Tubing Sealed in Packers[J]. Journal of Petroleum Technology, 1962, 14(6):655-670.

[3] Hammerlindl D J. Movement, Forces, and Stresses Associated With Combination Tubing Strings Sealed in Packers[J]. Journal of Petroleum Technology, 1977, 29(2):195-208.

[4] Hammerlindl D J. Packer-to-Tubing Forces for Intermediate Packers[J]. Journal of Petroleum Technology, 1980, 32(3):515-527.

[5] Qingdao Li, Guangping Xie, Juan Zhang. Calculation Analysis of Initial Amount of Compression of Test String_-The Third Discussion of Force Analsis System of Packer String[J]. Drilling \& Production Technology, 2001, 24(6):48-51.

[6] Qingdao Li, Guangping Xie, Juan Zhang. Force Analysis of Permanent Packer String_ The Fifth Discussion of Force Analsis System of Packer String[J]. Drilling \& Production Technology, 2002, 25(2):61-65.
[7] Yihua Dou, Fuxiang Zhang. Mechanical Analysis of Well Testing Downhole String in Deep Well With HTHP and Its Application[J]. Drilling \& Production Technology, 2007,(05):17-20+26+164.

[8] Qi Liu, Jianxun Jiang, Qing Shi. Calculation Analysis on Force Deformation of Packer String with Free Movement[J]. Fault Block Oil and Gas Field, 2007, 14(1):61-63.

[9] Pengbo Dong, Yihua Dou. Analysis of Buckling Configuration and Restraint Forces of Tubular Strings Sealed in Parkers[J]. Oil Field Equipment, 2007, 36(10):14-17.

[10] Yuhua Liao, Bin Yang, Ming Li. Mechanic Model Analyses on Packer Pipe String Effect[J]. Mechanical, 2012(s1):40-43.

[11] Zhanguo Lv. Mechanics and Safety Analysis of Multi - packer String in Horizontal Wells[D]. Xi'an Shiyou University, 2014.

[12] Zhilun Xu. Simple Teaching of Elasticity[M]. Higher Education Press, 2013.

[13] Hongwen Liu, Jianxin Lin, Manling Cao. Material Mechanics[M]. Higher Education Press, 2011. 